\title{
"Les éditions EMH représentent la voix du corps médical suisse»
}

\section{Interview: Bruno Kesseli}

Dr med. et lic. phil., rédacteur en chef

\author{
Il y a près d'un an, Hans Kurt a pris les fonctions de président du conseil d'adminis- \\ tration des Editions médicales suisses EMH, qui comptent le Bulletin des médecins \\ suisses parmi leurs produits phare. Il présente un bilan intermédiaire, sous forme \\ d'interview.
}

Hans Kurt, en juin dernier, vous avez remplacé Ludwig T. Heuss au poste de président du conseil d'administration des Editions médicales suisses EMH. Vous étiez déjà membre du conseil d'administration depuis plusieurs années, en tant que représentant de la FMH. Qu'est-ce qui vous a motivé à prendre le relais de votre prédécesseur?

Durant mon mandat de membre du conseil d'administration, j'ai perçu les Editions EMH comme une entreprise passionnante, en pleine évolution. Au conseil d'administration comme au comité directeur, des spécialistes intéressés et engagés de la médecine et de l'édition travaillent en coopération. L'ambiance est très amicale, bienveillante. J'aime ce type de coopération, qui rend très créatif et génère une grande estime mutuelle.

Vous êtes maintenant président du conseil d'administration depuis près d'un an. Qu'est-ce que ces nouvelles fonctions ont changé pour vous?

En tant que président, je me sens plus responsable, d'une part envers la FMH, dont je suis en fin de compte le représentant élu, d'autre part, et surtout, envers l'entreprise EMH.

\section{Pouvez-vous préciser?}

Les EMH sont une entreprise de 20 personnes, très appréciée, qui doit pouvoir se développer dans l'avenir. Avec son directeur et les responsables du comité directeur, j'essaie d'intensifier les contacts avec la FMH, mais aussi avec d'autres partenaires, par exemple les éditions de Suisse romande Médecine et Hygiène. Je voudrais positionner les EMH comme une entreprise de médias, mettant des prestations médiatiques à la disposition de tous les médecins, en répondant davantage aux besoins des sociétés cantonales de médecine, des sociétés de discipline médicale ou des autres organisations du secteur médical.

«Les Editions EMH sont plus qu'un des nombreux acteurs du marché des éditions médicales, elles représentent la voix du corps médical suisse."

Les Editions EMH sont spécifiques à plusieurs égards. En discutant avec vos collègues, on constate que les représentations de la forme de l'entreprise, donc du "concept EMH" qu'elle couvre, sont très vagues. En tant que président du conseil d'adminis-
Dr med. Hans Kurt

Le Dr med. Hans Kurt a grandi à Soleure et a fait ses études de médecine à l'université de Fribourg (Nuithonie) et à Berne. Il exerce depuis plus de 25 ans comme psychiatre et psychothérapeute dans un cabinet médical de groupe interdisciplinaire à Soleure (www.grup penpraxis-weststadt.ch - en allemand). II est formé en thérapie systémique et supervision. II exerce différents mandats au sein d'associations ayant trait à la politique de la santé: il est depuis de longues années président et membre du Comité de la Société Suisse de psychiatrie et psychothérapie SSPP et est président de I'Alliance Santé Psychique Suisse; il a été viceprésident du conseil d'administration de Solothurner Spitäler AG jusqu'en 2014, est membre du conseil d'administration des Editions médicales suisses EMH depuis 2009, et membre du conseil transitoire des Services psychiatriques universitaires de Berne depuis 2014.

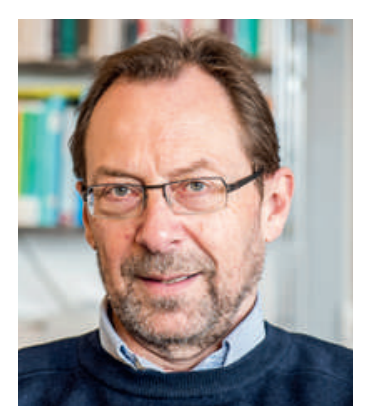


tration, pouvez-vous nous donner ici quelques précisions?

Ce qui fait la spécificité des EMH, c'est que sa création est le résultat de la réunion de deux partenaires très différents, il y a dix-huit ans de cela: d'une part, la FMH, qui détient la majorité des actions jusqu'à maintenant, d'autre part, la société Schwabe AG, riche d'une longue tradition dans le domaine des publications scientifiques et des produits éditoriaux de grande qualité. Cependant, Les EMH sont une entreprise indépendante, ce n'est pas simplement une organisation subordonnée à la FMH. C'est en ce sens que les Editions médicales suisses EMH doivent aussi mener leur propre politique d'entreprise, notamment pour pouvoir s'imposer comme leader du marché très concurrentiel des publications médicales.

\section{Dans quelle mesure les Editions EMH se dis- tinguent-elles des autres éditions médicales?} Les Editions EMH sont plus qu'un des nombreux acteurs du marché des éditions médicales, elles représentent la voix du corps médical suisse, ce qui peut parfois être difficile face à la concurrence. Il ne faut pas oublier que, même si la FMH détient la majorité des actions et occupe la présidence du conseil d'administration, l'entreprise est dirigée par deux partenaires égaux en droits.

\section{Que signifie pour vous «difficile face à la concur- rence»?}

En matière de politique éditoriale, les EMH observent une stricte indépendance par rapport aux annonceurs et aux bailleurs de fonds. Les Editions veulent observer une éthique de publication et attachent de l'importance à d'autres principes, à savoir être un employeur respectant des valeurs sociales et fabriquant ses produits en Suisse, dans la mesure du possible conformément à des critères écologiques.

\section{Le modèle que vous venez d'esquisser correspond-il encore aux exigences de l'époque? Pourquoi a-t-on encore besoin des EMH?}

Que ce soit pour des raisons de santé publique ou de politique professionnelle, le corps médical, donc la

\section{«En matière de politique éditoriale, les EMH observent une stricte indépendance par rapport aux annonceurs et aux bailleurs de fonds."}

FMH, dépendent de la publication de produits médiatiques indépendants. Et il ne s'agit pas seulement de revues, mais aussi de plus en plus d'offres issues des médias électroniques. La FMH n'ayant pas elle-même les compétences ni les ressources dans le domaine de l'édition et de l'impression, elle a besoin d'un partenaire fiable, qui d'une part recueille l'estime des professionnels, d'autre part peut garantir un niveau de qualité élevé.

Depuis la fondation des EMH, les temps ont changé à plusieurs égards, même dans le milieu des publications médicales.

L'an prochain les Editions EMH fêteront leur vingtième anniversaire. L'article 2 des statuts de 1997 stipule que l'objectif de la société par actions doit être la publication de produits médiatiques dans le domaine médical. A ce jour, rien n'a changé sur ce point. Mais la forme des produits médiatiques et le comportement des lecteurs se sont transformés ces dernières années. On a certes encore besoin d'articles bien documentés, et scientifiquement irréprochables, mais aussi d'informations brèves, type 20 minutes, de blogs de discussions et d'un accès rapide à des questions précises, avec des liens. Sans oublier le bon vieux facteur d'impact, qui touche la concurrence, par exemple via les métriques alternatives comme «altmetrics» et «Google Scholar».

Quels sont les atouts spécifiques à l'entreprise et dans quels domaines voyez-vous les plus belles opportunités pour les EMH?

La légèreté de sa structure et l'important potentiel de ses collaborateurs comptent certainement parmi les atouts des EMH. Tous les collaborateurs des EMH que j'ai

«La mutation rapide du secteur de la publication et les bouleversements dans les médias exigent une évolution permanente des offres existantes et un état d'esprit ouvert à la nouveauté.»

rencontrés jusque-là sont parfaitement qualifiés, passionnés par leur travail et s'identifient fortement à l'entreprise. En outre, grâce à leurs produits de grande qualité, les Editions EMH sont leader sur le marché suisse des publications médicales, comme le montrent les enquêtes correspondantes. Le fait que les Editions EMH soient fondées sur une coopération entre la FMH et Schwabe garantit leur prestige dans l'opinion publique.

\section{Quels sont pour vous les principaux défis et risques?}

Je pense qu'à l'avenir nous devrons affronter deux défis différents: la transition des médias imprimés vers les médias électroniques et le futur financement de médias indépendants. Les Editions EMH se sont rapidement imposées sur Internet et ont défendu un accès libre à 


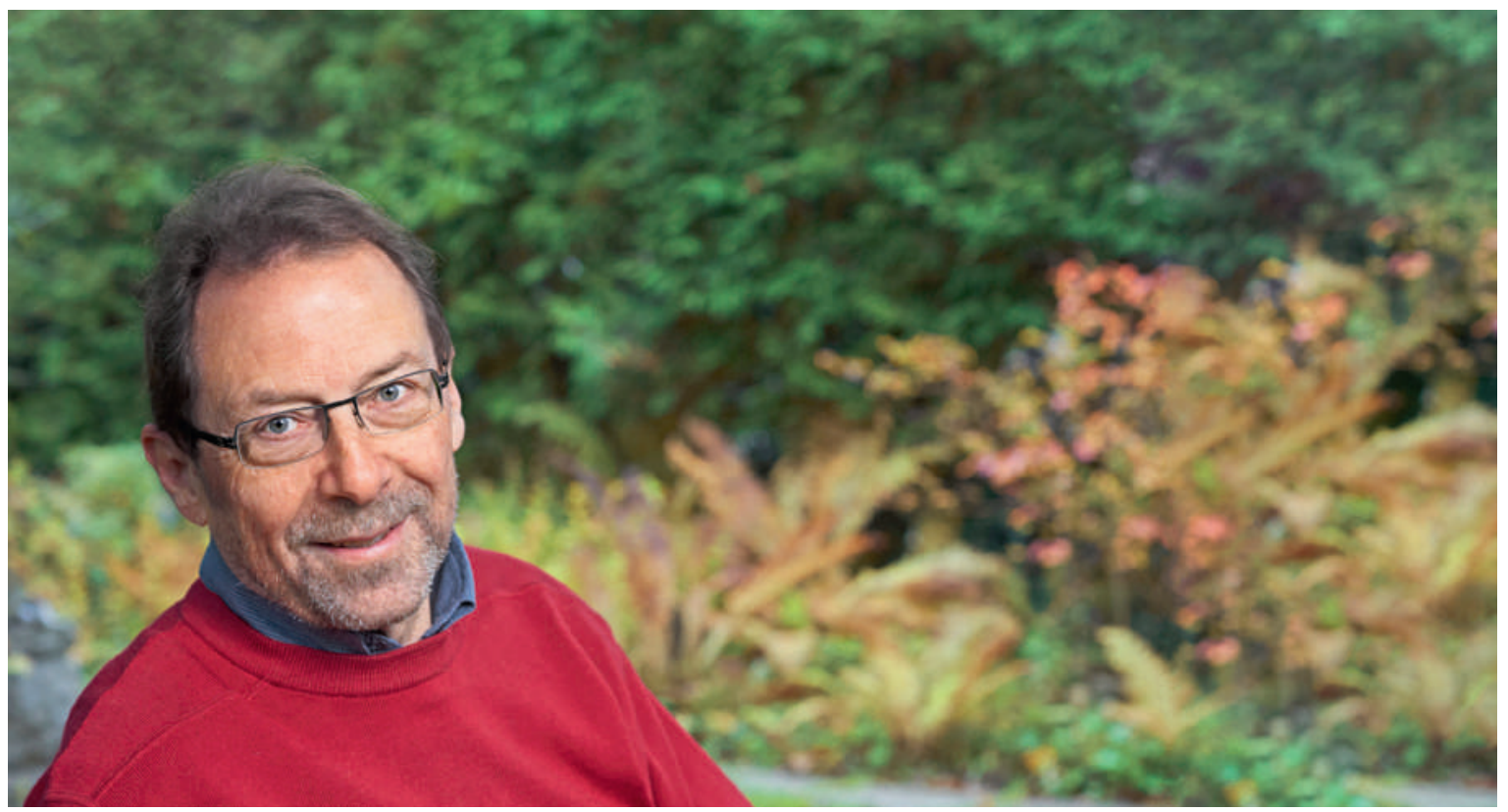

Hans Kurt: "En tant que président, je me sens plus responsable.»

leurs publications. La revue Swiss Medical Weekly (www. smw.ch) fut l'une des premières revues médicales à avoir son propre site Internet. Et cette revue en ligne et en accès libre illustre de façon exemplaire de nouvelles possibilités et difficultés. La mutation rapide du secteur de la publication et les bouleversements dans les médias exigent une évolution permanente des offres existantes et un état d'esprit ouvert à la nouveauté. Tout cela dans un contexte économique délicat, dans lequel on prône l'économie et où les sources de financement traditionnelles connaissent un fort recul.

\section{Alors comment financer les évolutions nécessaires?}

Dès à présent, les Editions EMH doivent être économes en moyens, tout en travaillant constamment à leur indépendance financière. Elles sont donc confrontées à la même pression budgétaire que la FMH, qui impose des exigences en conséquence. Malgré les défis qui s'annoncent et qui impliqueraient des besoins supplémentaires, les ressources financières ne sont pas plus importantes. Selon moi, à l'avenir, il ne sera plus possible de proposer des produits médiatiques attrayants et de bonne qualité sans demander un paiement, sous une forme quelconque (abonnement ou autre).

\section{Quelle importance future accordez-vous aux an-} nonces, qui financent jusqu'à maintenant l'essentiel des revues des $E M H$ ?

L'indépendance et un niveau de qualité élevé ne seront plus couverts dans les mêmes proportions par les an- nonces et les sponsors, comme ce fut le cas pendant longtemps. N'oublions pas que la FMH a profité pendant des années des excédents engrangés. Malheureusement ces temps sont révolus, suite au rétrécissement

«Les Editions EMH vont vraiment se positionner dans les nouveaux médias, mais avec des produits attrayants et de qualité.»

du marché des annonces. La forte dépendance des annonces, notamment dans le secteur de l'industrie pharmaceutique, mais aussi pour ce qui est du marché de l'emploi, représente donc un risque considérable pour le futur des EMH. Un engagement durable de la part des membres de la FMH, avec un abonnement adapté, permettrait de limiter les incertitudes qui reviennent tous les ans.

\section{Quelle stratégie appliquent les EMH par rapport à ce} qu'on appelle les nouvelles technologies, qui ne sont plus si nouvelles que ça mais se sont développées à une vitesse vertigineuse?

Les Editions EMH sont déjà en train de franchir différentes étapes dans l'univers des médias prétendument nouveaux. Tous nos produits imprimés ont désormais été modifiés et fabriqués de façon à être disponibles au format électronique, avec les gadgets correspondants. Mais il est impératif de s'imposer encore plus sur Internet. 


\section{A quoi pensez-vous?}

Par exemple à des offres de formation continue en ligne. Nous sommes aussi en discussion sur un site web, destiné au grand public, pour lequel il faudrait veiller à associer la FMH comme actionnaire principal, avec les produits et informations correspondants. Cependant, avec Swiss Medical Weekly, qui est déjà une revue en ligne, nous voyons bien à quel point il est difficile de trouver un financement pour de tels produits. Là aussi, nous devons inventer notre propre chemin pour trouver des possibilités de financement. Les Editions EMH vont vraiment se positionner dans les nouveaux médias, mais avec des produits attrayants et de qualité, pas seulement avec des présentations médiatiques bon marché, destinées à faire sensation.

Les avis sur l'importance future des médias imprimés - dans le cas des EMH, les revues imprimées divergent beaucoup dans la profession. Comment envisagez-vous l'avenir des produits EMH dans ce domaine?

Depuis des années, on prédit la disparition des médias imprimés. Pourtant, de nombreux lecteurs continuent d'apprécier la lecture du BMS ou du SMF sur papier. C'est aussi une question d'âge: les médecins plus jeunes vont souhaiter de plus en plus d'offres en ligne, tandis que le corps médical plus âgé - loin d'être négligeable privilégie la forme traditionnelle des revues. Nous pourrions maîtriser cette transition en complétant de plus en plus les rédactions avec de jeunes collègues identifiant plus tôt les besoins à prendre à compte.

En tant que président du conseil d'administration, où souhaitez-vous mettre personnellement l'accent dans les prochaines années? Quels objectifs vous tiennent particulièrement à cœur?

En fait, j'ai deux souhaits pour les prochaines années. Je voudrais déjà que la confiance du corps médical dans les Editions médicales suisses EMH augmente, et que le conseil d'administration et le comité directeur ne soient plus constamment confrontés aux préjugés et aux critiques injustifiés. Il est urgent que les Editions médicales suisses EMH puissent se concentrer sur leur cœur d'activité, après ces années de turbulences et les différents contrôles et analyses financiers. En tant que président du conseil d'administration, je voudrais consolider cette confiance et élaborer des conditions favorables au développement des Editions EMH. Cela suppose aussi que nous accordions assez d'importance au bien-être des collaboratrices et collaborateurs.

«En tant que président du conseil d'administration, je voudrais consolider cette confiance et élaborer des conditions favorables au développement des Editions EMH.»

\section{Quel est votre second souhait?}

Ensuite, je souhaite instamment que les produits médiatiques des $\mathrm{EMH}$, quels qu'ils soient, restent indépendants et respectent une éthique de publication. Il faut préserver la diversité des opinions du corps médical, mais aussi de la politique de la santé. Les Editions EMH ne souhaitent pas être influencées ni par l'industrie pharmaceutique, ni par les représentants d'intérêts, quels qu'ils soient.

Conclusion: Après une année à la tête des Editions, travailler dans le contexte indéniablement difficile des éditions médicales vous fait-il toujours plaisir? Resterez-vous encore un moment président du conseil d'administration des EMH?

Depuis toujours je trouve le travail chez les EMH intéressant. Bien sûr, depuis que je suis président du conseil d'administration, je le trouve aussi difficile. Je fus moi-même engagé auparavant dans les revues des Editions EMH, où j'ai découvert les ressources qu'il fallait déployer pour fabriquer et rédiger de bonnes revues médicales. Tout comme j'ai pris plaisir à rédiger, je prends plaisir à travailler chez les EMH, en coopération avec la FMH et les responsables de Schwabe. En un mot, je ne suis pas près de partir!

\footnotetext{
Crédits photo

Hans Kurt
} 